\title{
Infrared and NMR spectral Hammett correlations in 4-(2-naphthyl)-5,6-dihydro-6-(substituted phenyl)- ${ }^{4} H$ - 1,3-oxazine-2-amines
}

\author{
G. Thirunarayanan $^{1, *}$, S. Pazhamalai ${ }^{2}$, K. G. Sekar ${ }^{3}$ \\ ${ }^{1}$ Department of Chemistry, Annamalai University, Annamalainagar - 608 002, India \\ ${ }^{2}$ DDE Wing of Chemistry, Annamalai University, Annamalainagar - 608 002, India \\ ${ }^{3}$ Department of Chemistry, National College, Tiruchirappalli - 620001, India \\ E-mail address: drgtnarayanan@gmail.com
}

\begin{abstract}
A series containing ten titled compounds have been synthesised and recorded the IR and NMR spectra. From the spectra the infrared $v \mathrm{NH}, \mathrm{C}=\mathrm{N}, \mathrm{C}-\mathrm{O}-\mathrm{C}$ stretches, NMR chemical shifts of $(\delta, \mathrm{ppm})$ $\mathrm{NH}$ and $\mathrm{C}=\mathrm{N}$ were assigned and correlated with Hammett substituent constants, $\mathrm{F}$ and $\mathrm{R}$ parameters using single regression analysis. From the results, the effects of substituent on the above spectral frequencies were discussed.
\end{abstract}

Keywords: Oxazine-2-amines; IR and NMR spectra; Hammett equation; Correlation analysis

\section{INTRODUCTION}

Hammett equation is one of the best tools for predicting the effect of substituents on the reaction centre of substrates through kinetic parameter or spectral data with linear regression analysis [1]. The spectral parameter is used for studying the effect of substituent on the substrate and ground state equilibration such as $E$ and $Z$ isomers, s-cis and s-trans conformers, cis and gauche conformers [2-4]. Correlation analysis was utilized for studying the transition state of the reaction mechanism [5], enol-enone tautomerism [6], structureactivity relationships of bio active molecules [7] and electrochemical redox behaviour of compounds [8]. The unsaturated cyclic oxazine-2-amines are important bioactive molecules. They possess many biologically activities such as antimicrobial [9], anti-plasmodial [10], anti-cancer [11], anti-depressants [12], cytotoxicity [13], anti-osteoplastic [14], anti-tumour [15], anti-oxidant [16], anti-tuberculosis [17], anti-neoplastic [18], antagonists [19], antiinflammatory [20] anti-infectants [21], IKB kinase beta [22], PTP-1B inhibition [23] and insect antifeedant activities [24].

Recently, Thirunarayanan et al., have synthesised and evaluated the antimicrobial and insect antifeedant activities of some aryl oxazine derivatives [9,24]. The effect of substituent on the spectral group frequencies of some 4-(6-methoxy-2-naphthyl)-5,6-dihydro-6(substituted phenyl)- ${ }^{4} H-1,3$-oxazine-2-amines were studied by Thirunarayanan [25]. There is no report available for the study of effect of substituents on the spectral group frequencies of 
titled compounds in literature in the past. Therefore the authors have taken efforts to synthesized some 4-(2-naphthyl)-5,6-dihydro-6-(substituted phenyl)- ${ }^{4} H$-1,3-oxazine-2amines and recorded the infrared and nuclear magnetic resonance spectra for studying the Hammett spectral correlations.

\section{EXPERIMENTAL}

\section{1. General}

All chemicals and solvents used in this present study were procured from SigmaAldrich and Merck companies. The infrared spectra of all oxazine imines have been recorded in SHIMADUZ Fourier Transform IR spectrophotometer using $\mathrm{KBr}$ disc. The NMR spectra of all compounds were recorded in BRUKER AV 400 type spectrometer, using $\mathrm{CDCl}_{3}$ as a solvent, $400 \mathrm{MHz}$ frequency was applied for recording ${ }^{1} \mathrm{H}, 100 \mathrm{MHz}$ for ${ }^{13} \mathrm{C}$ NMR spectra, taking TMS as standard.

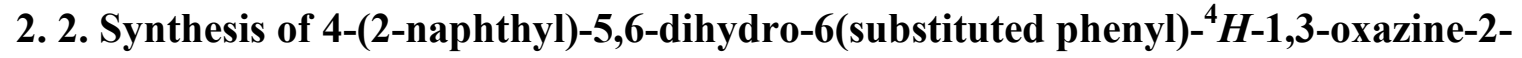 amines}

The titled oxazine-2-amines were synthesized and their purities were checked by literature method [26]. Substituted styryl 2-naphthyl ketones $(2 \mathrm{mmol})$, urea $(2 \mathrm{mmol})$ and 0.5 $\mathrm{g}$ of fly-ash: $\mathrm{H}_{2} \mathrm{SO}_{4}$ were subjected to microwave irradiation for 2-4 $\mathrm{m}$ at $650 \mathrm{~W}$ (Scheme 1) (Samsung, Microwave Oven, 100-700 W). Dichloromethane $(10 \mathrm{~mL})$ was added to the reaction-production mixture after completion of reaction, followed by simple filtration. Concentration of the extract afforded the crude product and this was purified by recrystallization with ethanol.

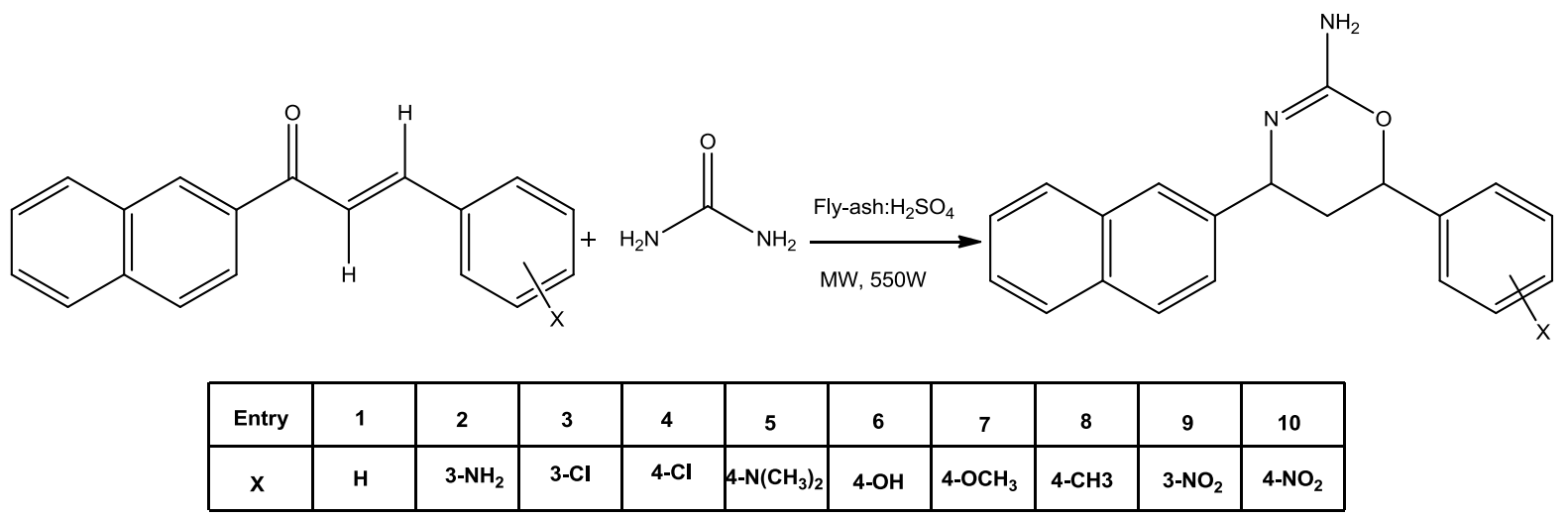

Scheme 1. Synthesis of 4-(2-naphthyl)-5,6-dihydro-6-(substituted phenyl)- ${ }^{4} H$-1,3-oxazine-2-amines by fly-ash: $\mathrm{H}_{2} \mathrm{SO}_{4}$ catalyzed cyclization of aryl chalcones and urea under microwave irradiation.

\section{RESULTS AND DISCUSSION}

In the present study, the author have investigated the effect of substituents on the infrared $v \mathrm{NH}, \mathrm{C}=\mathrm{N}, \mathrm{C}-\mathrm{O}-\mathrm{C}\left(\mathrm{cm}^{-1}\right)$ stretches, ${ }^{1} \mathrm{H}$ NMR $\delta \mathrm{NH},{ }^{13} \mathrm{C} \mathrm{NMR} \delta \mathrm{C}=\mathrm{N}(\mathrm{ppm})$ chemical shifts of 4-(2-naphthyl)-5,6-dihydro-6(substituted phenyl)- ${ }^{4} H$-1,3-oxazine-2-amines by Hammett correlation. 


\section{1. Infrared spectral study}

In infrared spectral study, the $v \mathrm{NH}, \mathrm{C}=\mathrm{N}$ and $\mathrm{C}-\mathrm{O}-\mathrm{C}\left(\mathrm{cm}^{-1}\right)$ stretches of synthesized amines were correlated by the Hammett equation as shown in equation (1).

$$
v=\rho \sigma+v_{0}
$$

where $v_{0}$ is the frequency for the parent member of the series.

The assigned $v \mathrm{NH}, \mathrm{C}=\mathrm{N}\left(\mathrm{cm}^{-1}\right)$ stretches, ${ }^{1} \mathrm{H}$ NMR $\delta \mathrm{NH},{ }^{13} \mathrm{C} \quad \mathrm{NMR} \delta \mathrm{C}=\mathrm{N}(\mathrm{ppm})$ chemical shifts of 4-(2-naphthyl)-5,6-dihydro-6(substituted phenyl)- ${ }^{4} H$-1,3-oxazine-2-amines were presented in Table1. These data were correlated with Hammett substituent constants, F and $\mathrm{R}$ parameters linear regression analysis [1-4,8,27-29]. The result of statistical analysis was presented in Table 2 .

Table 1. The infrared $v \mathrm{NH}, \mathrm{C}=\mathrm{N}, \mathrm{C}-\mathrm{O}-\mathrm{C}\left(\mathrm{cm}^{-1}\right)$ stretches, ${ }^{1} \mathrm{H}$ NMR $\delta \mathrm{NH},{ }^{13} \mathrm{C}$ NMR $\delta \mathrm{C}=\mathrm{N}(\mathrm{ppm})$ chemical shifts of 4-(2-naphthyl)-5,6-dihydro-6-(substituted phenyl) ${ }^{4} H$-1,3-oxazine-2-amines.

\begin{tabular}{|c|c|c|c|c|c|c|}
\hline \multirow{2}{*}{ Entry } & \multirow{2}{*}{$\mathrm{X}$} & \multicolumn{3}{|c|}{$\mathrm{IR}\left(\nu, \mathrm{cm}^{-1}\right)$} & ${ }^{1} \mathrm{H}(\delta, \mathrm{ppm})$ & ${ }^{13} \mathrm{C}(\delta, \mathrm{ppm})$ \\
\cline { 3 - 7 } & & $\mathrm{NH}$ & $\mathrm{C}=\mathrm{N}$ & $\mathrm{C}-\mathrm{O}-\mathrm{C}$ & $\mathrm{NH}$ & $\mathrm{C}=\mathrm{N}$ \\
\hline 10 & $\mathrm{H}$ & 3526 & 1598 & 1215 & 2.291 & 165.02 \\
\hline 11 & $3-\mathrm{NH}_{2}$ & 3548 & 1592 & 1210 & 2.112 & 165.25 \\
\hline 12 & $3-\mathrm{Cl}$ & 3545 & 1605 & 1215 & 2.225 & 164.25 \\
\hline 13 & $4-\mathrm{Cl}$ & 3535 & 1589 & 1222 & 2.125 & 165.28 \\
\hline 14 & $4-\mathrm{N}(\mathrm{CH})_{2}$ & 3552 & 1621 & 1214 & 2.091 & 164.57 \\
\hline 15 & $4-\mathrm{OH}^{2}$ & 3545 & 1596 & 1221 & 2.204 & 165.33 \\
\hline 16 & $4-\mathrm{OCH}_{3}$ & 3532 & 1613 & 1215 & 2.220 & 164.72 \\
\hline 17 & $4-\mathrm{CH}_{3}$ & 3535 & 1622 & 1221 & 2.212 & 164.47 \\
\hline 18 & $3-\mathrm{NO}_{2}$ & 3555 & 1625 & 1215 & 2.321 & 165.41 \\
\hline 19 & $4-\mathrm{NO}_{2}$ & 3555 & 1648 & 1217 & 2.182 & 165.32 \\
\hline
\end{tabular}

The correlation of infrared $v \mathrm{NH}\left(\mathrm{cm}^{-1}\right)$ stretches of 4-(2-naphthyl)-5,6-dihydro-6(substituted phenyl)- ${ }^{4} H-1,3$-oxazine-2-amines were satisfactorily with Hammett $\sigma, \sigma^{+}, \sigma_{\mathrm{I}}$ constants and $\mathrm{F}$ parameters excluding $3-\mathrm{NH}_{2}$ and $4-\mathrm{N}\left(\mathrm{CH}_{3}\right)_{2}$. When these substituents were included in the correlation, the correlation was reduced considerably. The Hammett $\sigma_{R}$ constant and $\mathrm{R}$ parameter were fail in correlation. This is due to the inability of transmittance of effect of substituent on the group frequencies and associated with resonance-conjugative structure as shown in Figure 1. All correlations gave positive $\rho$ values. This implies that the normal substituent effects operates in all systems. 


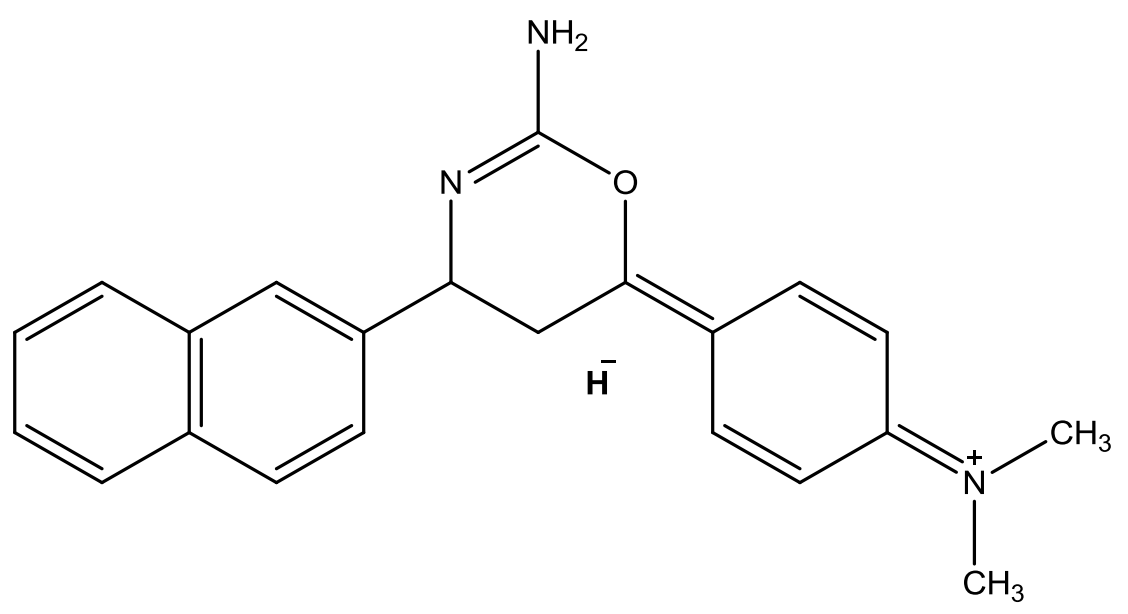

Figure 1. The resonance-conjugative structure.

The correlation of infrared $\mathrm{vCN}\left(\mathrm{cm}^{-1}\right)$ stretches of 4-(2-naphthyl)-5,6-dihydro-6(substituted phenyl)- ${ }^{4} H-1,3$-oxazine-2-amines were satisfactorily with Hammett $\sigma, \sigma^{+}$and $\sigma_{\mathrm{R}}$ constants excluding 4-N( $\left(\mathrm{CH}_{3}\right)_{2}$ substituent. The Hammett $\sigma_{\mathrm{I}}$ constant, $\mathrm{F}$ and $\mathrm{R}$ parameters have shown poor correlations. All correlations gave positive $\rho$ values. This implies that the normal substituent effect operates in all systems. The failure in correlation was due to the reason stated earlier and associated with the conjugative-structure as shown in Figure 1.

Table 2. Results of statistical analysis of IR, ${ }^{1} \mathrm{H}$ NMR and ${ }^{13} \mathrm{C}-\mathrm{NMR}$ spectral values of 4-(2naphthyl)-5,6-dihydro-6-(substituted phenyl)- ${ }^{4} H-1,3$-oxazine-2-amines with Hammett $\sigma, \sigma^{+}, \sigma_{\mathrm{I}}, \sigma_{\mathrm{R}}$ constants, $\mathrm{F}$ and $\mathrm{R}$ parameters.

\begin{tabular}{|c|c|c|c|c|c|c|c|}
\hline Frequency & Constant & $\mathbf{r}$ & $\mathbf{I}$ & $\rho$ & $\mathbf{s}$ & $\mathbf{n}$ & Correlated derivatives \\
\hline \multirow{6}{*}{ 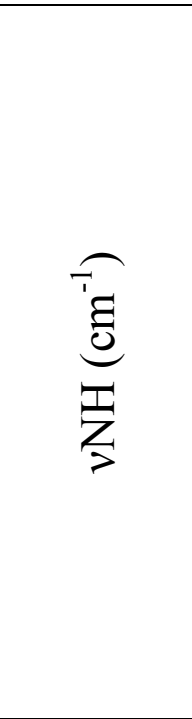 } & $\sigma$ & 0.903 & 3542.35 & 7.359 & 10.18 & 10 & 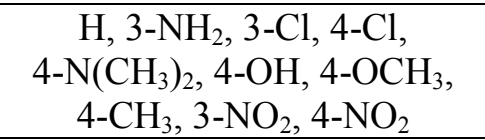 \\
\hline & $\sigma^{+}$ & 0.901 & 3543.16 & 1.999 & 10.71 & 10 & $\begin{array}{c}\mathrm{H}, 3-\mathrm{NH}_{2}, 3-\mathrm{Cl}, 4-\mathrm{Cl} \\
4-\mathrm{N}\left(\mathrm{CH}_{3}\right)_{2}, 4-\mathrm{OH}, 4-\mathrm{OCH}_{3} \\
4-\mathrm{CH}_{3}, 3-\mathrm{NO}_{2}, 4-\mathrm{NO}_{2}\end{array}$ \\
\hline & $\sigma_{\mathrm{I}}$ & 0.905 & 3546.70 & 21.009 & 9.20 & 8 & $\begin{array}{c}\mathrm{H}, 3-\mathrm{Cl}, 4-\mathrm{Cl}, 4-\mathrm{OH}, 4-\mathrm{OCH}_{3}, \\
4-\mathrm{CH}_{3}, 3-\mathrm{NO}_{2}, 4-\mathrm{NO}_{2}\end{array}$ \\
\hline & $\sigma_{\mathrm{R}}$ & 0.805 & 3543.28 & 2.344 & 10.81 & 10 & $\begin{array}{c}\mathrm{H}, 3-\mathrm{NH}_{2}, 3-\mathrm{Cl}, 4-\mathrm{Cl} \\
\text { 4- } \mathrm{N}\left(\mathrm{CH}_{3}\right)_{2}, 4-\mathrm{OH}, 4-\mathrm{OCH}_{3} \\
\text { 4- } \mathrm{CH}_{3}, 3-\mathrm{NO}_{2}, 4-\mathrm{NO}_{2}\end{array}$ \\
\hline & $\mathrm{F}$ & 0.905 & 3535.04 & 24.663 & 8.81 & 8 & $\begin{array}{c}\mathrm{H}, 3-\mathrm{Cl}, 4-\mathrm{Cl}, 4-\mathrm{OH}, 4-\mathrm{OCH}_{3}, \\
4-\mathrm{CH}_{3}, 3-\mathrm{NO}_{2}, 4-\mathrm{NO}_{2}\end{array}$ \\
\hline & $\mathrm{R}$ & 0.807 & 3542.15 & 1.976 & 1080 & 10 & $\begin{array}{c}\mathrm{H}, 3-\mathrm{NH}_{2}, 3-\mathrm{Cl}, 4-\mathrm{Cl} \\
\text { 4-N(}\left(\mathrm{CH}_{3}\right)_{2}, 4-\mathrm{OH}, 4-\mathrm{OCH}_{3} \\
\text { 4- } \mathrm{CH}_{3}, 3-\mathrm{NO}_{2}, 4-\mathrm{NO}_{2}\end{array}$ \\
\hline \multirow{3}{*}{ 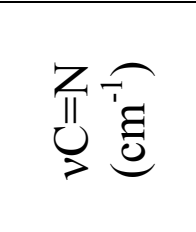 } & $\sigma$ & 0.903 & 1610.03 & 14.132 & 18.24 & 9 & $\begin{array}{l}\mathrm{H}, 3-\mathrm{NH}_{2}, 3-\mathrm{Cl}, 4-\mathrm{Cl}, 4-\mathrm{OH}, \\
4-\mathrm{OCH}_{3}, 4-\mathrm{CH}_{3}, 3-\mathrm{NO}_{2}, 4-\mathrm{NO}_{2}\end{array}$ \\
\hline & $\sigma^{+}$ & 0.902 & 1611.88 & 5.372 & 19.06 & 9 & $\begin{array}{l}\mathrm{H}, 3-\mathrm{NH}_{2}, 3-\mathrm{Cl}, 4-\mathrm{Cl}, 4-\mathrm{OH}, \\
4-\mathrm{OCH}_{3}, 4-\mathrm{CH}_{3}, 3-\mathrm{NO}_{2}, 4-\mathrm{NO}_{2}\end{array}$ \\
\hline & $\sigma_{\mathrm{I}}$ & 0.803 & 1603.59 & 2.194 & 18.33 & 10 & $\mathrm{H}, 3-\mathrm{NH}_{2}, 3-\mathrm{Cl}, 4-\mathrm{Cl}$ \\
\hline
\end{tabular}




\begin{tabular}{|c|c|c|c|c|c|c|c|}
\hline & & & & & & & $\begin{array}{c}\text { 4- } \mathrm{N}\left(\mathrm{CH}_{3}\right)_{2}, 4-\mathrm{OH}, 4-\mathrm{OCH}_{3}, \\
\text { 4- } \mathrm{CH}_{3}, 3-\mathrm{NO}_{2}, 4-\mathrm{NO}_{2}\end{array}$ \\
\hline & $\sigma_{\mathrm{R}}$ & 0.904 & 1618.05 & 34.379 & 17.10 & 9 & $\begin{array}{c}\mathrm{H}, 3-\mathrm{NH}_{2}, 3-\mathrm{Cl}, 4-\mathrm{Cl} \\
\text { 4-OH, 4-OCH }, 4-\mathrm{CH}_{3} \\
\text { 3- } \mathrm{NO}_{2}, 4-\mathrm{NO}_{2}\end{array}$ \\
\hline & $\mathrm{F}$ & 0.804 & 1600.85 & 33.493 & 17.61 & 10 & $\begin{array}{c}\mathrm{H}, 3-\mathrm{NH}_{2}, 3-\mathrm{Cl}, 4-\mathrm{Cl}, \\
4-\mathrm{N}\left(\mathrm{CH}_{3}\right)_{2}, 4-\mathrm{OH}, 4-\mathrm{OCH}_{3}, \\
4-\mathrm{CH}_{3}, 3-\mathrm{NO}_{2}, 4-\mathrm{NO}_{2}\end{array}$ \\
\hline & $\mathrm{R}$ & 0.883 & 1616.45 & 16.942 & 18.27 & 10 & $\begin{array}{c}\mathrm{H}, 3-\mathrm{NH}_{2}, 3-\mathrm{Cl}, 4-\mathrm{Cl}, \\
\left.\text { 4-N(} \mathrm{CH}_{3}\right)_{2}, 4-\mathrm{OH}, 4-\mathrm{OCH}_{3}, \\
\text { 4- } \mathrm{CH}_{3}, 3-\mathrm{NO}_{2}, 4-\mathrm{NO}_{2}\end{array}$ \\
\hline & $\sigma$ & 0.817 & 1216.56 & 1.278 & 4.00 & 10 & $\begin{array}{c}\mathrm{H}, 3-\mathrm{NH}_{2}, 3-\mathrm{Cl}, 4-\mathrm{Cl}, \\
\left.\text { 4-N( } \mathrm{CH}_{3}\right)_{2}, 4-\mathrm{OH}, 4-\mathrm{OCH}_{3}, \\
\text { 4- } \mathrm{CH}_{3}, 3-\mathrm{NO}_{2}, 4-\mathrm{NO}_{2}\end{array}$ \\
\hline & $\sigma^{+}$ & 0.808 & 1216.50 & 0.042 & 4.00 & 10 & 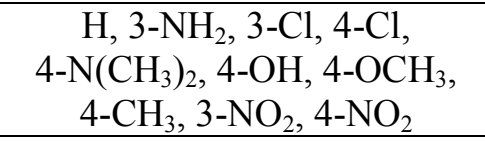 \\
\hline 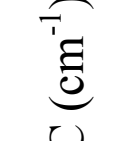 & $\sigma_{\mathrm{I}}$ & 0.810 & 1216.06 & 1.501 & 3.98 & 10 & $\begin{array}{c}\mathrm{H}, 3-\mathrm{NH}_{2}, 3-\mathrm{Cl}, 4-\mathrm{Cl}, \\
\left.\text { 4- } \mathrm{N}^{-} \mathrm{CH}_{3}\right)_{2}, 4-\mathrm{OH}, 4-\mathrm{OCH}_{3}, \\
\text { 4- } \mathrm{CH}_{3}, 3-\mathrm{NO}_{2}, 4-\mathrm{NO}_{2}\end{array}$ \\
\hline$\bigcup_{\substack{1 \\
0}}^{1}$ & $\sigma_{\mathrm{R}}$ & 0.821 & 1217.14 & 3.122 & 3.91 & 10 & $\begin{array}{c}\mathrm{H}, 3-\mathrm{NH}_{2}, 3-\mathrm{Cl}, 4-\mathrm{Cl}, \\
\left.\text { 4-N(} \mathrm{CH}_{3}\right)_{2}, 4-\mathrm{OH}, 4-\mathrm{OCH}_{3}, \\
\text { 4- } \mathrm{CH}_{3}, 3-\mathrm{NO}_{2}, 4-\mathrm{NO}_{2}\end{array}$ \\
\hline & $\mathrm{F}$ & 0.852 & 1215.79 & 2.341 & 3.96 & 10 & $\begin{array}{c}\mathrm{H}, 3-\mathrm{NH}_{2}, 3-\mathrm{Cl}, 4-\mathrm{Cl}, \\
\text { 4- }\left(\mathrm{CH}_{3}\right)_{2}, 4-\mathrm{OH}, 4-\mathrm{OCH}_{3}, \\
4-\mathrm{CH}_{3}, 3-\mathrm{NO}_{2}, 4-\mathrm{NO}_{2}\end{array}$ \\
\hline & $\mathrm{R}$ & 0.872 & 1217.75 & 2.365 & 3.88 & 10 & 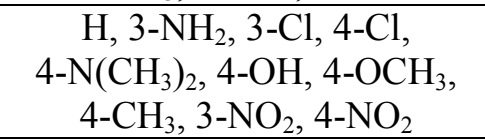 \\
\hline \multirow{6}{*}{ 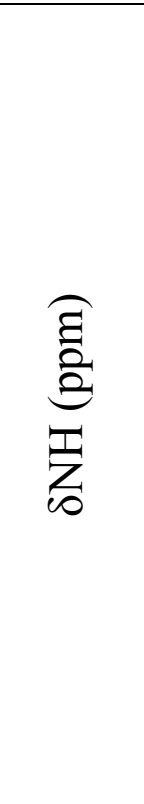 } & $\sigma$ & 0.904 & 2.194 & 0.066 & 0.05 & 10 & $\begin{array}{c}\mathrm{H}, 3-\mathrm{NH}_{2}, 3-\mathrm{Cl}, 4-\mathrm{Cl}, \\
\left.\text { 4- } \mathrm{N}^{-} \mathrm{CH}_{3}\right)_{2}, 4-\mathrm{OH}, 4-\mathrm{OCH}_{3} \\
\text { 4- } \mathrm{CH}_{3}, 3-\mathrm{NO}_{2}, 4-\mathrm{NO}_{2}\end{array}$ \\
\hline & $\sigma^{+}$ & 0.947 & 2.206 & 0.045 & 0.05 & 10 & $\begin{array}{c}\mathrm{H}, 3-\mathrm{NH}_{2}, 3-\mathrm{Cl}, 4-\mathrm{Cl}, \\
\text { 4-N(}\left(\mathrm{CH}_{3}\right)_{2}, 4-\mathrm{OH}, 4-\mathrm{OCH}_{3}, \\
\text { 4- } \mathrm{CH}_{3}, 3-\mathrm{NO}_{2}, 4-\mathrm{NO}_{2}\end{array}$ \\
\hline & $\sigma_{\mathrm{I}}$ & 0.920 & 2.176 & 0.063 & 0.06 & 10 & $\begin{array}{c}\mathrm{H}, 3-\mathrm{NH}_{2}, 3-\mathrm{Cl}, 4-\mathrm{Cl}, \\
\text { 4- } \mathrm{N}^{-}\left(\mathrm{CH}_{3}\right)_{2}, 4-\mathrm{OH}, 4-\mathrm{OCH}_{3}, \\
\text { 4- }-\mathrm{CH}_{3}, 3-\mathrm{NO}_{2}, 4-\mathrm{NO}_{2}\end{array}$ \\
\hline & $\sigma_{R}$ & 0.965 & 2.236 & 0.185 & 0.05 & 10 & $\begin{array}{c}\mathrm{H}, 3-\mathrm{NH}_{2}, 3-\mathrm{Cl}, 4-\mathrm{Cl}, \\
\text { 4-N(}\left(\mathrm{CH}_{3}\right)_{2}, 4-\mathrm{OH}, 4-\mathrm{OCH}_{3}, \\
\text { 4- } \mathrm{CH}_{3}, 3-\mathrm{NO}_{2}, 4-\mathrm{NO}_{2}\end{array}$ \\
\hline & $\mathrm{F}$ & 0.802 & 2.178 & 0.067 & 0.08 & 10 & $\begin{array}{c}\mathrm{H}, 3-\mathrm{NH}_{2}, 3-\mathrm{Cl}, 4-\mathrm{Cl}, \\
\text { 4-N(}\left(\mathrm{CH}_{3}\right)_{2}, 4-\mathrm{OH}, 4-\mathrm{OCH}_{3}, \\
\text { 4- } \mathrm{CH}_{3}, 3-\mathrm{NO}_{2}, 4-\mathrm{NO}_{2}\end{array}$ \\
\hline & $\mathrm{R}$ & 0.966 & 2.239 & 0.125 & 0.04 & 10 & $\begin{array}{c}\mathrm{H}, 3-\mathrm{NH}_{2}, 3-\mathrm{Cl}, 4-\mathrm{Cl} \\
\text { 4-N(}\left(\mathrm{CH}_{3}\right)_{2}, 4-\mathrm{OH}, 4-\mathrm{OCH}_{3} \\
\text { 4- } \mathrm{CH}_{3}, 3-\mathrm{NO}_{2}, 4-\mathrm{NO}_{2}\end{array}$ \\
\hline \multirow{2}{*}{$\bigcup_{\infty}^{\pi}$} & $\sigma$ & 0.904 & 164.94 & 0.349 & 0.41 & 10 & $\begin{array}{c}\mathrm{H}, 3-\mathrm{NH}_{2}, 4-\mathrm{Cl}, 4-\mathrm{N}\left(\mathrm{CH}_{3}\right)_{2}, \\
\text { 4-OH, 4-OCH }, 4-\mathrm{CH}_{3}, 3-\mathrm{NO}_{2}, \\
4-\mathrm{NO}_{2}\end{array}$ \\
\hline & $\sigma^{+}$ & 0.903 & 164.99 & 0.180 & 0.42 & 10 & $\begin{array}{c}\mathrm{H}, 3-\mathrm{NH}_{2}, 4-\mathrm{Cl}, 4-\mathrm{N}\left(\mathrm{CH}_{3}\right)_{2} \\
\text { 4-OH, 4-OCH } 3,4-\mathrm{CH}_{3}\end{array}$ \\
\hline
\end{tabular}




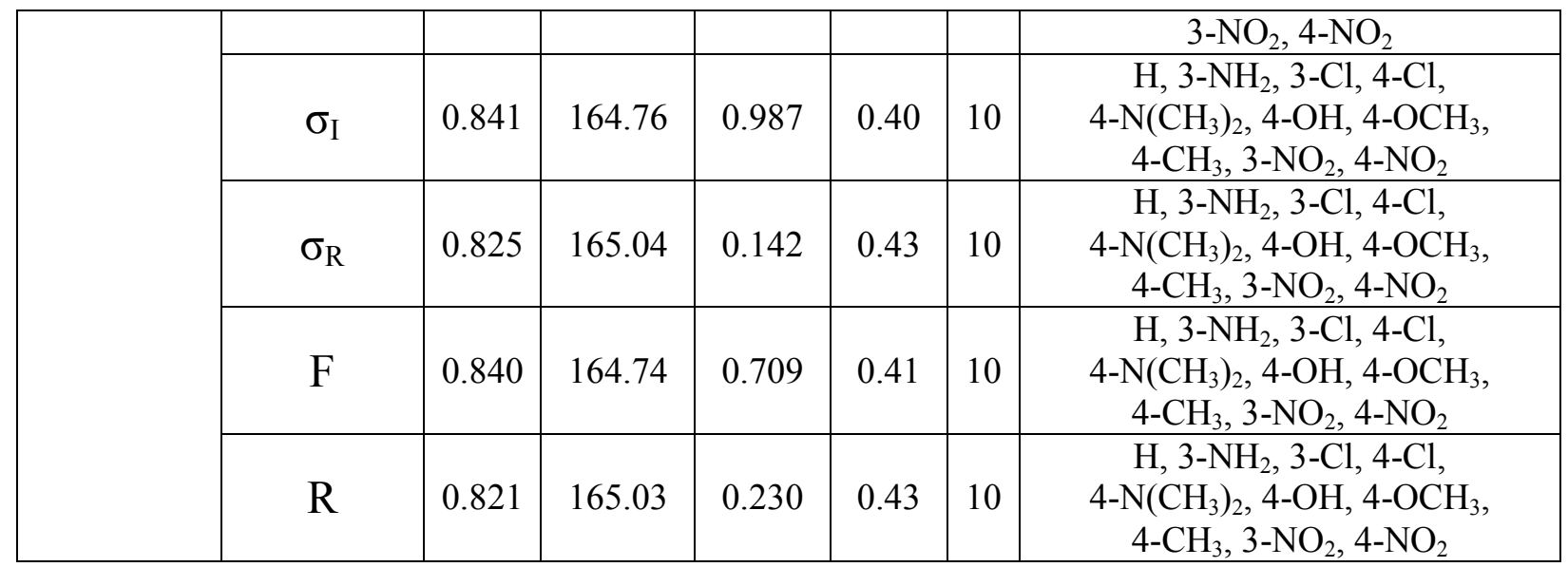

$\mathrm{r}=$ correlation coefficient $\mathrm{I}=$ intercept $\rho=$ slope; $\mathrm{s}=$ standard deviation; $\mathrm{n}=$ number of correlated derivatives

The correlation of infrared $v \mathrm{C}-\mathrm{O}-\mathrm{C}\left(\mathrm{cm}^{-1}\right)$ stretches of 4-(2-naphthyl)-5,6-dihydro-6(substituted phenyl)- ${ }^{4} H-1,3$-oxazine-2-amines were shown poor $\mathrm{r}$ values with Hammett substituent constants, $\mathrm{F}$ and $\mathrm{R}$ parameters. All correlations gave positive $\rho$ values. This implies that the normal substituent effects operate in all systems. The failure in correlation was due to the reason stated earlier and associated with the conjugative-structure as shown in Figure 1.

Some of the infrared stretches were fail in correlation with Hammett constants, F and R parameters in single linear regression analysis. They are worthwhile when seeking in multi parameter correlation with $\sigma_{I}$ and $\sigma_{R}$ or $F$ and R Swain-Lupton [30] parameters. The generated multi-regression analysis equations are given in (2-7).

$$
\begin{gathered}
\begin{array}{r}
v \mathrm{NH}\left(\mathrm{cm}^{-1}\right)=3532.88( \pm 6.864)+27.022( \pm 14.285) \sigma_{\mathrm{I}}+11.428( \pm 1.406) \sigma_{\mathrm{R}} \\
(\mathrm{r}=0.958, \mathrm{n}=10, \mathrm{P}>95 \%)
\end{array} \\
\begin{array}{c}
v \mathrm{NH}\left(\mathrm{cm}^{-1}\right)=3529.03( \pm 6.193)+33.222( \pm 12.980) \mathrm{F}+11.482( \pm 1798) \mathrm{R} \\
(\mathrm{r}=0.969, \mathrm{n}=10, \mathrm{P}>95 \%)
\end{array} \\
\begin{array}{r}
v \mathrm{C}=\mathrm{N}\left(\mathrm{cm}^{-1}\right)=1614.20( \pm 13.228)+9.708( \pm 2.753) \sigma_{\mathrm{I}}+29.431( \pm 2.709) \sigma_{\mathrm{R}} \\
(\mathrm{r}=0.949, \mathrm{n}=10, \mathrm{P}>90 \%)
\end{array} \\
\begin{array}{r}
v \mathrm{C}=\mathrm{N}\left(\mathrm{cm}^{-1}\right)=1606.06( \pm 13.791)+26.394( \pm 2.840) \mathrm{F}+0.413( \pm 1.777) \mathrm{R} \\
(\mathrm{r}=0.947, \mathrm{n}=10, \mathrm{P}>90 \%) \\
v \mathrm{C}-\mathrm{S}-\mathrm{C}\left(\mathrm{cm}^{-1}\right)=1217.22( \pm 3.078)+0.192( \pm 0.001) \sigma_{\mathrm{I}}+3.220( \pm 0.625) \sigma_{\mathrm{R}} \\
(\mathrm{r}=0.921, \mathrm{n}=10 \mathrm{P}>90 \%) \\
v \mathrm{C}-\mathrm{S}-\mathrm{C}\left(\mathrm{cm}^{-1}\right)=1216.98( \pm 3.100)+0.742( \pm 0.064) \mathrm{F}+2.153( \pm 0.399) \mathrm{R} \\
(\mathrm{r}=0.924, \mathrm{n}=10, \mathrm{P}>90 \%)
\end{array}
\end{gathered}
$$

\section{2. NMR spectral study}

In nuclear magnetic resonance spectra, the ${ }^{1} \mathrm{H}$ or the ${ }^{13} \mathrm{C}$ chemical shifts $(\delta)(\mathrm{ppm})$ depend on the electronic environment of the nuclei concerned. These chemical shifts have 
been correlated with reactivity parameters. Thus the Hammett equation may be used in the form as shown in (8).

$$
\log \delta=\log \delta_{0}+\rho \sigma
$$

where $\delta_{0}$ is the chemical shift of the corresponding parent compound.

The assigned ${ }^{1} \mathrm{H}$ NMR $\delta \mathrm{NH},{ }^{13} \mathrm{C}$ NMR $\delta \mathrm{C}=\mathrm{N}$ (ppm) chemical shifts of 4-(2-naphthyl)5,6-dihydro-6-(substituted phenyl)- ${ }^{4} H$-1,3-oxazine-2-amines were presented in Table 1. These data were correlated with Hammett substituent constants, F and R parameters linear regression analysis [1-4,8,27-29]. The result of statistical analysis was presented in Table 2. From Table 2, the $\delta \mathrm{NH}$ (ppm) chemical shifts of 4-(2-naphthyl)-5,6-dihydro-6-(substituted phenyl) ${ }^{4} H-1,3$-oxazine-2-amines were satisfactorily correlated with Hammett substituent constants and $\mathrm{R}$ parameters. The Field effect of the substituents were fail in correlation and it unable to predict the reactivity on the $\mathrm{NH}$ chemical shifts. This is due to the reason stated earlier and associated with Figure 1. All correlations gave positive $\rho$ values. This means that then normal substituent effects operates in all systems.

From Table 2, the ${ }^{13} \mathrm{C}$ NMR $\delta \mathrm{C}=\mathrm{N}(\mathrm{ppm})$ chemical shifts of 4-(2-naphthyl)-5,6dihydro-6-(substituted phenyl)- ${ }^{4} H$-1,3-oxazine-2-amines were satisfactorily correlated with Hammett $\sigma$ and $\sigma^{+}$constants produce satisfactory correlations excluding 3-Cl substituent. The Hammett $\sigma_{\mathrm{I}}, \sigma_{\mathrm{R}}$ substituent constants, $\mathrm{F}$ and $\mathrm{R}$ parameters were fail in correlation. This is due to the inability of substituent constants for prediction of reactivity on the chemical shifts of oxazine amines and associated with resonance conjugative structure shown in Fig. 1. All correlations gave positive $\rho$ values. This means that then a normal substituent effect operates in all systems.

In view of the inability of prediction of effect of substituents by single regression analysis with Hammett substituent constants, $\mathrm{F}$ and $\mathrm{R}$ parameters, they are worthwhile when seeking in multi-regression analysis for ${ }^{1} \mathrm{H} N M R \quad \delta \mathrm{NH},{ }^{13} \mathrm{C} N \mathrm{NR} \delta \mathrm{C}=\mathrm{N}(\mathrm{ppm})$ chemical shifts of 4-(6-methoxy-2-naphthyl)-5,6-dihydro-6-(substituted phenyl)- ${ }^{4} H$-1,3-oxazine-2amines. The generated multi-regression [30] analysis equations are given in (9-12):

$$
\begin{gathered}
\delta \mathrm{NH}(\mathrm{ppm})=2.255( \pm 0.045)+0.046( \pm 0.002) \sigma_{\mathrm{I}}+0.208( \pm 0.094) \sigma_{\mathrm{R}} \\
(\mathrm{r}=0.966, \mathrm{n}=10, \mathrm{P}>95 \%) \\
\delta \mathrm{NH}(\mathrm{ppm})=2.253( \pm 0.046)+0.035( \pm 0.002) \mathrm{F}+0.136( \pm 0.060) \mathrm{R} \\
(\mathrm{r}=0.967, \mathrm{n}=10, \mathrm{P}>95 \%) \\
\delta \mathrm{C}=\mathrm{N}(\mathrm{ppm})=164.79( \pm 0.317)+0.634( \pm 0.066) \sigma_{\mathrm{I}}+0.098( \pm 0.006) \sigma_{\mathrm{R}} \\
(\mathrm{r}=0.941, \mathrm{n}=10, \mathrm{P}>90 \%) \\
\delta \mathrm{C}=\mathrm{N}(\mathrm{ppm})=164.76( \pm 0.327)+0.682( \pm 0.068) \mathrm{F}+0.036( \pm 0.004) \mathrm{R} \\
(\mathrm{r}=0.940, \mathrm{n}=10, \mathrm{P}>90 \%)
\end{gathered}
$$

\section{CONCLUSIONS}

Totally ten 4-(2-naphthyl)-5,6-dihydro-6-(substituted phenyl)- ${ }^{4} H-1,3$-oxazine-2-amine compounds were synthesized and recorded IR and NMR spectra. The infrared $v \mathrm{NH}, \mathrm{C}=\mathrm{N}, \mathrm{C}-$ $\mathrm{O}-\mathrm{C}\left(\mathrm{cm}^{-1}\right)$ stretches, ${ }^{1} \mathrm{H}$ NMR $\delta \mathrm{NH},{ }^{13} \mathrm{C} \mathrm{NMR} \delta \mathrm{C}=\mathrm{N}(\mathrm{ppm})$ chemical shifts of synthesized 
oxazine amines were assigned and correlated with Hammett substituent constants, $\mathrm{F}$ and $\mathrm{R}$ parameters. From the single parameter correlation analyses, the infrared $v \mathrm{NH}, \mathrm{C}=\mathrm{N}\left(\mathrm{cm}^{-1}\right)$ stretches and $\mathrm{NMR} \delta \mathrm{NH}$ and $\delta \mathrm{C}=\mathrm{N}(\mathrm{ppm})$ chemical shifts correlated satisfactorily with Hammett substituent constants, $\mathrm{F}$ and $\mathrm{R}$ parameters. All the above spectral frequencies were satisfactorily correlated with $\sigma_{I}$ and $\sigma_{R}$ or $F$ and R Swain-Lupton parameters in multiregression analysis.

\section{References}

[1] G. Thirunarayanan, International Letters of Chemistry, Physics and Astronomy 9(2) (2013) 152-161.

[2] S. Vijayakumar, et al., International Letters of Chemistry, Physics and Astronomy 9(1) (2013) 68-86.

[3] G. Thirunarayanan, M. Gopalakrishnan, G. Vanangamudi, Spectrochim. Acta 67A (2007) 1106-1612.

[4] G. Thirunarayanan, G. Vanangamudi, V. Sathiyendiran, K. Ravi, Indian J. Chem. 50B(4) (2011) 593-604.

[5] G. K. Dass, Indian J. Chem. 40(A)(1) (2001) 23-29.

[6] Y. H. Wang, J. W. Zou, B. Zhang, Y. X. Lu, H. X. Jin, Q. S. Yu, J. Mol. Struct. (Theochem) 755(1-2) (2005) 31-37.

[7] V. Horváth, Z. Varga and A. Kovács, J. Mol. Struct. (Theochem) 755(1-3) (2005) 247-251.

[8] D. Kamalakkannan, G. Vanangamudi, R. Arulkumaran, K. Thirumurthy, P. Mayavel, G. Thirunarayanan, Elixir Org. Chem. 46 (2012) 8157-8166.

[9] G. Thirunarayanan, R. Arulkumaran, R. Sundararajan, International Letters of Chemistry, Physics and Astronomy 4 (2014) 82-97.

[10] B. P. Mathew, A. Kumar, S. Sharma, P. K. Shukla, M. Nath, Euro. J. Med. Chem. 45 (2010) 1502-1507.

[11] M. J. Elarfi, H. A. Al-Difar, Sci. Rev.Chem. Commun. 2(2) (2012) 103-107.

[12] V. Tiwari, J. Meshram, P. Ali, J. Sheikh, U. Tripathi, J. Enzyme Inhibit. Medi. Chem. 26(4) (2011) 569-578.

[13] B. C. Das, A. V. Madhukumar, J. Anguiano, S. Mani, Bioorg. Med. Chem. Lett. 19(15) (2009) 4204-4206.

[14] D. Zhou, B. L. Harrison, U. Shah, T. H. Andree, G. A. Hornby, R. Scerni, Bioorg. Med. Chem. Lett. 16(5) (2006) 1338-1341.

[15] S. Wang, Y. Li, Y. Liu, A. Lu, Q. You, Bioorg. Med. Chem. Lett. 18(14) (2008) 4095-4097.

[16] Y. Ando, K. Ando, M. Yamaguchi, J. Kunitomo, M. Koida, R. Fukuyama, Bioorg. Med. Chem. Lett. 16(22) (2006) 5849-5854. 
[17] L. Benameur, Z. Bouaziz, P. Nebois, M. H. Bartoli, M. Boitard, H. Fillion, Chem. Pharm. Bull. 44(3) (1996) 605-608.

[18] K. Roy, I. Mitra, A. Saha, Chem. Biol. Drug Design 74(5) (2009) 507-516.

[19] A. Blaser, D. Palmer, S. H. Sutherland, I. Kmentova, S. G. Franzblau, B. Wan, J. Med. Chem. 55(1) (2012) 312-326.

[20] L. Seal, D. Von Hoff. R. Lawrence, E. Izbicka, R. M. Jamison, Invest. New Drug 15(4) (1997) 289-296.

[21] B. Brudeli, L. R. Moltzau, K. W. Andressen, K. A. Krobert, J. Klaveness, F. O. Levy, Bioorg. Med. Chem. Lett. 18(24) (2010) 8600-8613.

[22] M. Akhter, A. Husain, N. Akhter, M. S.Y. Khan, Indian J. Pharm. Sci. 73 (2011) 101-104.

[23] D. Gothi, J. M. Joshi, Recent Patent. Antiinfect. Drug Discovery 6(1) (2011) 27-37.

[24] G. Thirunarayanan, V. Renuka, K. G. Sekar, K. Lakshmanan, K. Anbarasu, International Letters of Chemistry, Physics and Astronomy 4 (2014) 66-81.

[25] G. Thirunarayanan, International Letters of Chemistry, Physics and Astronomy 4 (2014) 109-116.

[26] G. Thirunarayanan, K. G. Sekar, Der Pharma Chemica 5(6) (2013)142-148.

[27] G. Thirunarayanan, International Letters of Chemistry, Physics and Astronomy 5 (2014) 89-98.

[28] G. Thirunarayanan, M. Suresh, International Letters of Chemistry, Physics and Astronomy 4 (1) (2014) 1-11.

[29] G. Thirunarayanan, K. G. Sekar, J. Saudi Chem. Soc. (2013);

DOI: $10.1016 /$ j.jscs.2013.12.002.

[30] C. G. Swain, E. C. Lupton Jr, J. Am. Chem. Soc. 90 (1968) 4324-4337. 